\title{
Coherent structural relaxation of water from meso- to intermolecular scales measured using neutron spectroscopy with polarization analysis
}

\author{
Arantxa Arbe $\odot,{ }^{1}$ Gøran J. Nilsen $\odot,{ }^{2}$ J. Ross Stewart $\odot,{ }^{2}$ Fernando Alvarez $\odot,{ }^{1,3}$ \\ Victoria García Sakai ${ }^{\circ}{ }^{2}$ and Juan Colmenero ${ }^{1,3,4, *}$ \\ ${ }^{1}$ Centro de Física de Materiales (CFM) (CSIC-UPV/EHU)-Materials Physics Center (MPC), Paseo Manuel de Lardizabal 5 , \\ 20018 San Sebastián, Spain \\ ${ }^{2}$ ISIS Neutron and Muon Source, Rutherford Appleton Laboratory, Didcot, OX11 OQX, United Kingdom \\ ${ }^{3}$ Departamento de Física de Materiales (UPV/EHU), Apartado 1072, 20080 San Sebastián, Spain \\ ${ }^{4}$ Donostia International Physics Center, Paseo Manuel de Lardizabal 4, 20018 San Sebastián, Spain
}

(Received 8 August 2019; accepted 16 March 2020; published 17 April 2020)

\begin{abstract}
By means of the recent implementation of neutron polarization analysis on a wide-angle time-of-flight spectrometer, we have been able to separately measure coherent and incoherent dynamic structure factors of heavy water with sub-meV resolution in a wide scattering vector $(Q)$ range. The observed decorrelation of collective fluctuations at mesoscales hardly depends on $Q$. In the crossover towards intermolecular scales, the coherent dynamics is nicely described by the convolution of a $Q$-independent mode and diffusion, in addition to collective excitations.
\end{abstract}

DOI: 10.1103/PhysRevResearch.2.022015

The collective dynamics of water in the terahertz range has been a topic of intense activity since the 1970s when the classic work of Rahman and Stillinger was published [1]. Most of the work following this focused on the double peak structure of the inelastic part of the spectrum of density fluctuations - the so-called, at the time, question of "fast" and "normal" sound dispersion in water. Although the first experimental investigations were carried out using inelastic neutron scattering [2,3], since the 1990s most studies were performed using inelastic x-ray scattering (IXS) (see the recent review, [4]). The current consensus is that the terahertz density fluctuations in water at the mesoscopic scale, and in particular the question of the acoustic propagation, can be explained in terms of a viscoelastic model (see [4], and references therein). This model assumes the existence of a "structural" relaxation process which should control the viscoelastic transition. The presence of such a relaxation implies that acoustic propagation in water will depend on how the sound-wave frequency compares with $1 / \tau$ where $\tau$ is the structural relaxation time. A direct observation by IXS of this relaxation process in the coherent spectra of water is difficult due to the current resolution of IXS $(\geqslant 1.5 \mathrm{meV})$. Values of $\tau$ of 1-2 ps at $300 \mathrm{~K}$ are usually deduced by modeling the IXS measurements by suitable viscoelastic equations, including, obviously, some assumptions. A simple Maxwell relationship also delivers viscoelastic times in the range of picoseconds [5]. Apart from a few exceptions [6,7] the IXS measurements

\footnotetext{
*juan.colmenero@ehu.eus

Published by the American Physical Society under the terms of the Creative Commons Attribution 4.0 International license. Further distribution of this work must maintain attribution to the author(s) and the published article's title, journal citation, and DOI.
}

are usually limited to mesoscopic scales (scattering vector $Q$ in the range $\left.0.2 \AA^{-1} \leqslant Q \leqslant 0.7 \AA^{-1}\right)$. Thus, the crossover towards the intermolecular scale $\left(Q \approx 2 \AA^{-1}\right.$ for water) where a "de Gennes-like" renormalization [8] is expected for the coherent structural relaxation, has not been well explored up to now.

Quasielastic neutron scattering (QENS) is the ideal technique with which to directly observe the structural relaxation of water in a wide $Q$ range, due to the high energy resolutions currently available. However, the problem is that the measured neutron-scattering (NS) intensity always contains a combination of coherent and incoherent contributions, corresponding to pair- and self-correlations, respectively. In the case of molecules like water with a high content of hydrogen atoms, the incoherent contribution, $I_{\text {inc }}$, is dominant due to the large incoherent NS cross section of ${ }^{1} \mathrm{H}$ nuclei $\left(\sigma_{\text {inc }}^{H} \approx 80\right.$ barn $)$. It is generally assumed that when replacing ${ }^{1} \mathrm{H}$ by deuterium, ${ }^{2} \mathrm{D}\left(\sigma_{\text {inc }}^{D} \approx \sigma_{\text {inc }}^{H} / 40\right)$, the scattering of (heavy) water becomes mainly coherent. However, this is only true at the maximum of the static structure factor, $S(Q)$, where the coherent contribution, $I_{\text {coh }}$, amounts to about six times $I_{\text {inc }}$ [see Fig. 1(a)]. At lower $Q$ values $I_{\text {coh }} \approx I_{\text {inc }}$, such that the total scattering is dominated by diffusive contributions [9]. Coherent and incoherent NS contributions can be unambiguously separated using uniaxial neutron polarization analysis (PA) [10]. Unfortunately, this technique poses many technical difficulties especially for wide-angle diffractometers and time-of-flight (TOF) spectrometers (see, e.g., [11,12]). Furthermore, the limited polarized neutron flux available limits both the available energy resolution and the statistical accuracy, and only static (diffraction) studies are routinely attempted [13-15]. Only recently have a few multidetector spectrometers demonstrated the capability of performing neutron spectroscopy with PA and sub-meV resolution [16-18]. In this work, we report on an experimental investigation of coherent structural relaxation 

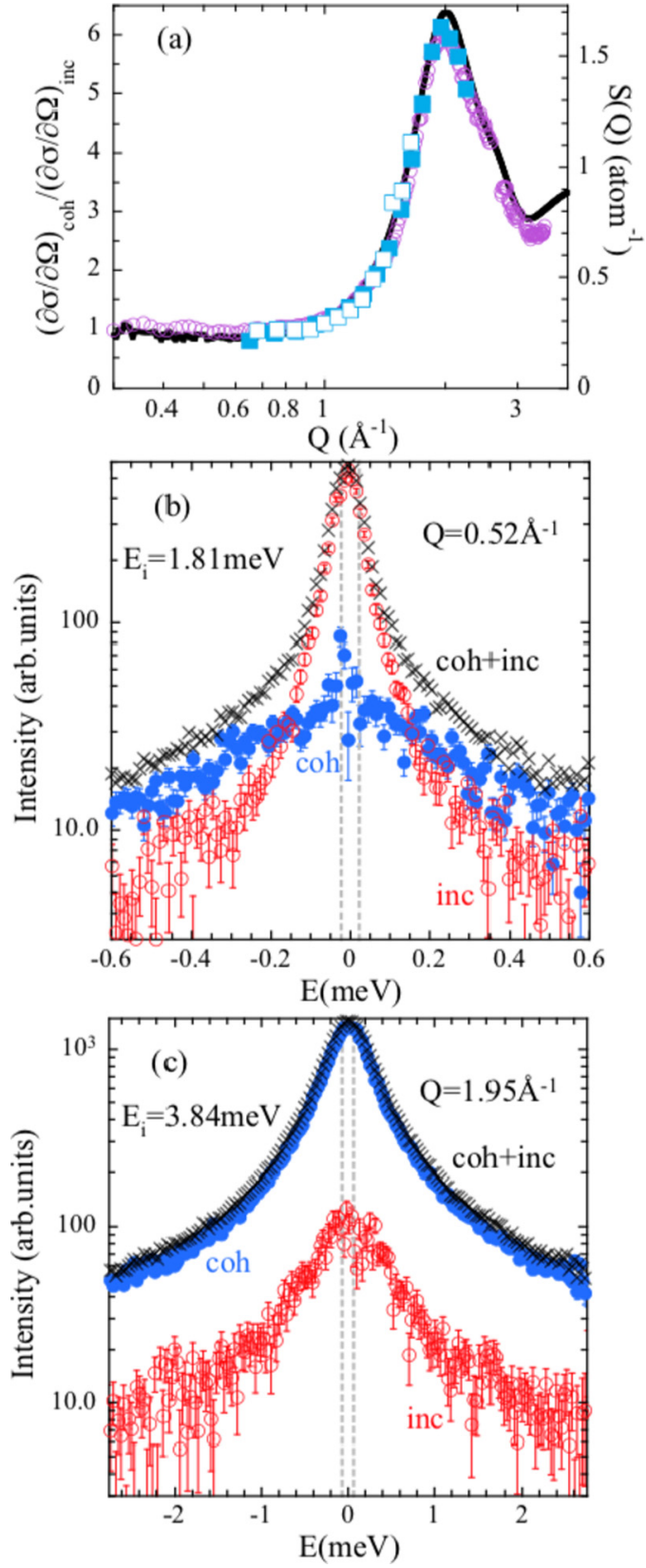

FIG. 1. (a) The ratio between coherent and incoherent differential cross sections $(\partial \sigma / \partial \Omega)_{\text {coh }} /(\partial \sigma / \partial \Omega)_{\text {inc }}$ of $\mathrm{D}_{2} \mathrm{O}$ at $298 \mathrm{~K}$ (hollow dots) as a function of $Q$ obtained by diffraction with PA (D7 at the Institute Laue Langevin, Grenoble, France) [9], and calculated from MD simulations (solid line, see SM [19]). The figure also includes the additional $y$-axis scale (right) corresponding to $S(Q)=$ $(\partial \sigma / \partial \Omega)_{\operatorname{coh}} /\left[\sigma_{\mathrm{coh}} /(4 \pi)\right]$ where $\sigma_{\mathrm{coh}} /(4 \pi)=\lim _{Q \rightarrow \infty}(\partial \sigma / \partial \Omega)_{\mathrm{coh}}$. Squares represent the QENS amplitudes (rescaled to match the D7 data) obtained from the application of the model to the LET results (filled, $E_{i}=3.84 \mathrm{meV}$; empty, $E_{i}=1.81 \mathrm{meV}$ ). (b) and (c) Comparison of the coherent and incoherent dynamical structure factors measured by LET on $\mathrm{D}_{2} \mathrm{O}$ at $295 \mathrm{~K}$ at the $Q$ values indicated, and the total spectra (coherent + incoherent). Vertical dotted lines represent the resolution FWHM.

of heavy water by means of QENS with uniaxial PA in a wide $Q$ range from meso- to intermolecular scales.
The experiments were carried out on the LET direct geometry TOF spectrometer at the ISIS Neutron and Muon Source, Oxfordshire, UK. Incident energies $E_{i}$ of $3.84,1.81$, and $1.05 \mathrm{meV}$ were selected by the chopper system running in an intermediate resolution setting, yielding full-width halfmaximum (FWHM) resolutions of $\Delta E=131 \mu \mathrm{eV}\left(3.4 \% E_{i}\right)$, $\Delta E=45 \mu \mathrm{eV}\left(2.5 \% E_{i}\right)$, and $\Delta E=22 \mu \mathrm{eV}\left(2.1 \% E_{i}\right)$, respectively, at the elastic line. Here, $E=h v$ is the energy transfer ( $h$ : Planck's constant). Polarization, flipping, and analysis of the beam were achieved using a supermirror polarizer, precession coil flipper, and hyperpolarized ${ }^{3} \mathrm{He}$ spin filter analyzer, respectively. The data collected with the flipper on and off were corrected for the finite polarizing efficiency of the instrument using a procedure detailed in the Supplemental Material (SM) [19], and the results combined to yield the coherent and incoherent dynamical structure factors $S_{\text {coh }}(Q, v)$ and $S_{\text {inc }}(Q, v)$. Three temperatures, 280, 295, and $350 \mathrm{~K}$, were investigated. Complementary molecular dynamics (MD) simulations have also been carried out (see SM [19]).

Figures 1(b) and 1(c) show the coherent and incoherent spectra measured for $\mathrm{D}_{2} \mathrm{O}$ at $295 \mathrm{~K}$ at the two $Q$ values indicated in the figure. In the case of $Q=0.52 \AA^{-1}$, the coherent contribution is very broad in comparison with the incoherent one. As this broadening is a measure of the inverse of the corresponding relaxation time (FWHM $\sim 1 / \tau)$, this tells us, without any fitting, that the coherent characteristic time $\tau_{c}$ is of the order of ten times smaller than the incoherent (diffusive) relaxation time. The latter is close to that deduced from the total (incoherent plus coherent) spectra that would have been measured if PA was not used. Since the relaxation time at $Q \approx 0.5 \AA^{-1}$ reported from measurements on $\mathrm{D}_{2} \mathrm{O}$ at $298 \mathrm{~K}$ without PA is $\approx 20$ ps [9], then $\tau_{c}$ should be $\approx 2$ ps. As expected, the situation is very different in the $Q$ range of the maximum of $S(Q), Q_{\max }$. Figure 1(c) shows that at $Q_{\max }=1.95 \AA^{-1}$, the total scattering almost coincides with the coherent contribution. This first simple qualitative comparison confirms that (i) PA is absolutely essential to obtain an accurate description of the coherent dynamic structure factor in the low $Q$ range below the maximum of $S(Q)$, even when using $\mathrm{D}_{2} \mathrm{O}$; and (ii) the expected values of the collective relaxation time in this $Q$ range are in the picosecond range.

The NS data have been analyzed in terms of the imaginary part of the corresponding susceptibility $\chi^{\prime \prime}(Q, v)$. This can be calculated as $\chi^{\prime \prime}(Q, v)=\pi S(Q, v) /[1+n(v)]$ where $n(v)=\left(e^{h v / k T}-1\right)^{-1}$ is the Bose occupation factor $(k$ : Boltzmann constant) [19]. This analysis of QENS data better distinguishes between the different processes involved in $S(Q, v)$ [9]. Figure 2 shows some representative constant- $Q$ cuts of $\chi_{\text {coh }}^{\prime \prime}(Q, v)$ at $295 \mathrm{~K}$. We shall consider $295 \mathrm{~K}$ as the showcase temperature in the following, and the thermal behavior will be discussed later.

The term "structural relaxation" is used with different meanings by diverse authors. Here, we will denote as structural relaxation the component of the coherent dynamic structure factor that is not of "vibrational" origin.

First we will focus on the mesoscopic low- $Q$ range $\left(Q \lesssim 0.7 \AA^{-1}\right)$. To avoid the influence of the vibrational contribution, we have restricted the fitting range to $v<$ $100 \mathrm{GHz}$. In this range, we have described $\chi_{\text {coh }}^{\prime \prime}(Q, v)$ by a single Lorentzian function $\chi_{\mathrm{coh}}^{\prime \prime}(Q, v) \propto 2 \pi \nu \tau_{c} /\left[1+\left(2 \pi \nu \tau_{c}\right)^{2}\right]$ 


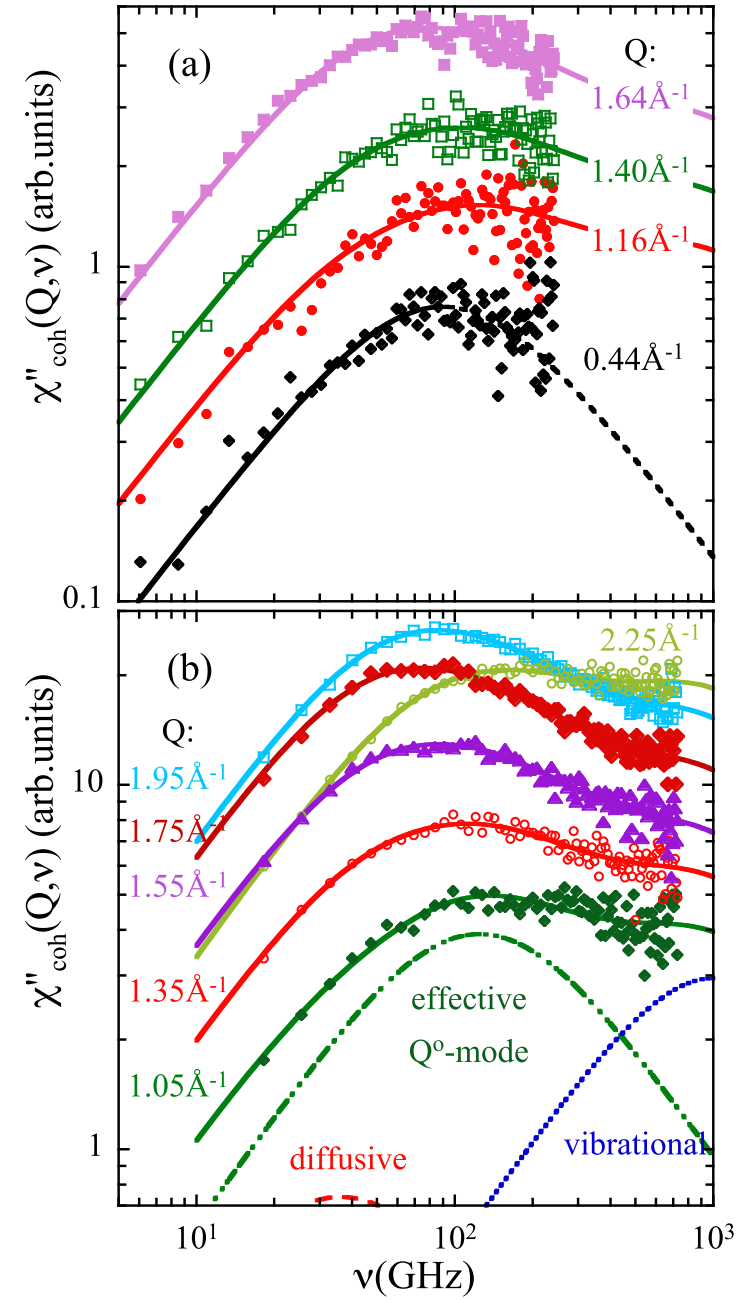

FIG. 2. Imaginary part of the dynamical susceptibility corresponding to the coherent dynamical structure factor of water at $295 \mathrm{~K}$ at the $Q$ values indicated. Results in (a) have been obtained with $E_{i}=1.81 \mathrm{meV}$ and in (b) with $E_{i}=3.84 \mathrm{meV}$. For $Q=0.44$ $\AA^{-1}$, the description in terms of a single Lorentzian up to $100 \mathrm{GHz}$ is shown by the solid line (extrapolated to higher frequencies by the dotted line). For the rest of the data, solid lines are fits with the proposed model. For $Q=1.05 \AA^{-1}$, the dashed, dashed-dotted, and dotted lines in (b) display the diffusive, effective $Q^{\circ}$-mode and vibrational contributions, respectively.

representing the relaxational contribution. The description of the experimental curves by this simple expression is rather good. An example of this kind of fitting is shown in Fig. 2(a) for $Q=0.44 \AA^{-1}$. The obtained coherent relaxation time $\tau_{c}(Q)$ displays little $Q$ dependence for the three temperatures investigated and ranges between 1.5 and 2 ps at $295 \mathrm{~K}$ [see Fig. 3(b)]. Our MD-simulation results at $300 \mathrm{~K}$ (see SM [19]) support the simple analysis carried out in this low- $Q$ range, confirming that (i) the vibrational and structural relaxational contributions are well separated; (ii) the vibrational contribution can be avoided at $v \lesssim 100 \mathrm{GHz}$; and (iii) the relaxational contribution can be well described by a single exponential decay (Lorentzian in the frequency domain) with an almost $Q$-independent characteristic time $\tau_{c}(Q)$ in the picosecond range.

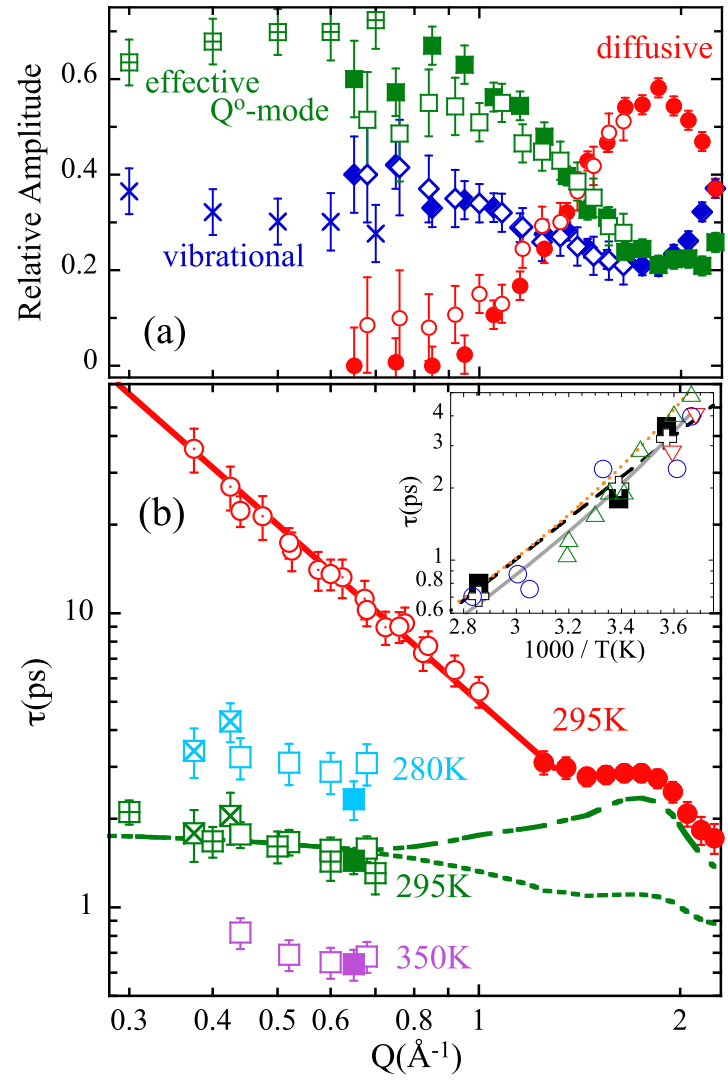

FIG. 3. (a) Relative contributions of the diffusive $[C(Q) A(Q)$, circles], effective $Q^{o}$ mode $[C(Q)[1-A(Q)]$, squares] and vibrational $\left[1-C(Q)\right.$, diamonds] processes to $\chi_{\mathrm{coh}}^{\prime \prime} / S(Q)$ at $T=295 \mathrm{~K}$ (filled symbols, $E_{i}=3.84 \mathrm{meV}$; empty symbols, $E_{i}=1.81 \mathrm{meV}$ ). MD-simulation results are also included $(\times$, vibrational; $\boxplus$, structural contributions). (b) $Q$ dependence of the characteristic relaxation times: $\tau_{d}$ (circles) and $\tau_{c}$ (squares). The $\tau_{d}$ values have been obtained from incoherent scattering $\left(\tau_{d}^{\text {inc }}\right)$ with $E_{i}=1.81 \mathrm{meV}(\bigcirc)$ and $E_{i}=$ $1.05 \mathrm{meV}(\odot)$, and from the fit of the coherent scattering results to the model (O), all at $295 \mathrm{~K}$. Solid line: fit of $D^{-1} Q^{-2}$ to $\tau_{d}^{\text {inc }}$, with $D=0.200 \pm 0.016 \AA^{2} / \mathrm{ps}$ in agreement with [24]. The $\tau_{c}$ values correspond to $E_{i}=1.81 \mathrm{meV}(\square), E_{i}=3.84 \mathrm{meV}(\boldsymbol{\square})$, and $E_{i}=$ $1.05 \mathrm{meV}(\otimes)$ obtained at $T=295$ (green), 280 (blue), and $350 \mathrm{~K}$ (magenta) and simulations $(\boxplus) . \tau_{c}$ and $\langle\tau\rangle$ values calculated with the model at $295 \mathrm{~K}$ are shown by the dashed and dashed-dotted lines, respectively. Inset: Arrhenius plot of $\tau_{c}^{o}(\boldsymbol{\square})$, the structural time from IXS (O) [4], Brillouin light scattering $(\nabla)$ [25] and Brillouin UV scattering $(\triangle)[26],\left\langle\tau\left(Q_{\max }\right)\right\rangle$ (crosses), and the laws describing the Arrhenius fit of $\tau_{c}^{o}$ (dashed line), the viscosity dependence $\eta / T$ (solid line), and the NMR rotational time for $\mathrm{D}_{2} \mathrm{O}$ [27] (dotted line).

Still in this low- $Q$ range $\left(Q \lesssim 0.7 \AA^{-1}\right)$, a similar analysis but now of $\chi_{\text {inc }}^{\prime \prime}(Q, v)$ and restricted to $v<50 \mathrm{GHz}$ (see SM [19]) delivers the diffusion time $\tau_{d}^{\text {inc }}(Q)=D^{-1} Q^{-2}$. Figure 3(b) shows that $\tau_{d}^{\text {inc }}(Q)$ is well separated from $\tau_{c}$. This indicates that diffusion does not play a role in the decay of collective correlations in this $Q$ range. Thus, the results obtained until now suggest the existence of a $Q$-independent coherent mode in the very low $Q$ range, in agreement with the recent finding of Sciortino et al. at $Q \leqslant 0.3 \AA^{-1}$ by simulations [28]. Following their terminology, we shall denote this mode as $Q^{o}$ mode, and call its characteristic time $\tau_{c}^{o}$. With 
increasing $Q, \tau_{d}^{\text {inc }}(Q)$ approaches $\tau_{c}(Q)$ [see Fig. 3(b)] and we can expect (i) some influence of diffusion on the above introduced $Q^{o}$ mode and (ii) the progressive opening of a new pure diffusive channel of density-density relaxations, that would dominate in the vicinity of the maximum of $S(Q)$.

To describe $\chi_{\text {coh }}^{\prime \prime}(Q, v)$ at $Q \gtrsim 0.7 \AA^{-1}$, where diffusion could start to play a role, we cannot apply the simple model (single Lorentzian function) used in the low- $Q$ range. We have applied a general model, which has been used previously to describe the merging of the $\alpha$ and $\beta$ processes in glass-forming polymers [29-31] and more recently to analyze the incoherent NS and NMR results of water [9,24]. This model considers a vibrational and a relaxational contribution. The latter consists of two processes, a diffusive and a $Q$ independent one, taking place simultaneously. Accordingly, the corresponding scattering functions in the frequency domain are combined with a convolution. The formulation of the model is simpler in the time domain, where the convolution becomes a product. In this domain, the function $F(Q, t)$, representing either the intermediate incoherent scattering function or the normalized dynamic structure factor $S(Q, t) / S(Q)$, is expressed as

$$
F(Q, t)=[1-C(Q)] F_{V}(Q, t)+C(Q) F_{R}(Q, t)
$$

with $F_{V}(Q, t)$ the vibrational contribution, weighted by the amplitude $[1-C(Q)]$, and $F_{R}(Q, t)$ the relaxational contribution, weighted by $C(Q) . F_{R}(Q, t)$ results from the combination of two independent processes: $F_{R}(Q, t)=F_{d}(Q, t) F_{c}(Q, t)$. Here the diffusive contribution is $F_{d}(Q, t)=e^{-t / \tau_{d}}$ with $\tau_{d}(Q)$ a diffusive time. $F_{c}(Q, t)$ is the $Q$-independent mode contribution (in our case, the $Q^{o}$-mode introduced above) parametrized as $F_{c}(Q, t)=A(Q)+[1-A(Q)] e^{-t / \tau_{c}^{o}}$ with amplitude $[1-$ $A(Q)]$ and $Q$-independent relaxation time $\tau_{c}^{o}$. Then, $F_{R}(Q, t)$ reads

$$
F_{R}(Q, t)=[1-A(Q)] e^{-t / \tau_{c}}+A(Q) e^{-t / \tau_{d}},
$$

where $\tau_{c} \equiv\left(\frac{1}{\tau_{c}^{o}}+\frac{1}{\tau_{d}}\right)^{-1}$ is a time characterizing the $Q$ independent mode affected by diffusion. Inserting Eq. (2) into Eq. (1) we obtain

$$
\begin{aligned}
F(Q, t)= & {[1-C(Q)] F_{V}(Q, t)+C(Q)[1-A(Q)] e^{-t / \tau_{c}} } \\
& +C(Q) A(Q) e^{-t / \tau_{d}} .
\end{aligned}
$$

Taking into account that the imaginary part of the susceptibility corresponding to a Debye function with characteristic time $\tau$ is $2 \pi \nu \tau /\left[1+(2 \pi \nu \tau)^{2}\right]$ and the expression for a damped resonance term for the vibrational contribution [3], we can write

$$
\begin{aligned}
\chi^{\prime \prime}(Q, v) \propto & {[1-C(Q)] \frac{v_{o}^{2} v\left(k_{o} / 2 \pi\right)}{\left[\left(v_{o}^{2}-v^{2}\right)^{2}+\left(v k_{o} / 2 \pi\right)^{2}\right]} } \\
& +C(Q)[1-A(Q)] \frac{2 \pi v \tau_{c}}{\left[1+\left(2 \pi v \tau_{c}\right)^{2}\right]} \\
& +C(Q) A(Q) \frac{2 \pi v \tau_{d}}{\left[1+\left(2 \pi v \tau_{d}\right)^{2}\right]} .
\end{aligned}
$$

Here, $v_{o}$ is the frequency and $k_{o}$ is the damping coefficient of the damped resonance. The values of the low-frequency sound dispersion for water are basically constant for $Q \geqslant 0.7 \AA^{-1}$ [4]; for $\mathrm{D}_{2} \mathrm{O}, v_{o}=1.75 \mathrm{THz}$ and $k_{o}=22.5 \mathrm{THz}$.
Before applying the model at $Q \gtrsim 0.7 \AA^{-1}$, we note that it is perfectly consistent with the analysis of the low- $Q$ results previously presented. We recall that there the diffusion time $\tau_{d}=\tau_{d}^{\text {inc }}=D^{-1} Q^{-2}$ is very large and hence we do not expect a direct contribution from diffusion in the relaxation of density-density correlations. Then, in the framework of the proposed model the single Lorentzian function previously used to fit the low-frequency part of $\chi_{\mathrm{coh}}^{\prime \prime}(Q, v)$ is now identified with the $\tau_{c}$-Lorentzian term and thus in the low- $Q$ range the deduced time $\tau_{c}(Q)$ equals $\left(D Q^{2}+1 / \tau_{c}^{o}\right)^{-1}$. This law nicely describes both the experimental and MD-simulation results [see Fig. 3(b)], leading to $\tau_{c}^{o}=1.8 \pm 0.2$ ps at $295 \mathrm{~K}$. Once we have determined the value of $\tau_{c}^{o}$, we are in the position to apply the model for $Q \gtrsim 0.7 \AA^{-1}$. Imposing this value of $\tau_{c}^{o}$ and the incoherent diffusive time $\tau_{d}=D^{-1} Q^{-2}$, all parameters of the model are fixed apart from the two amplitudes, $A(Q)$ and $C(Q)$, plus a total amplitude factor, which should follow $S(Q)$. This approach reproduces the experimental results very well for $Q$ values up to $Q \approx 1.2 \AA^{-1}$. At higher $Q$ values, approaching the $S(Q)$ peak, the description of the spectra requires considering a kind of $S(Q)$ modulation of the diffusive relaxation time, as expected from a de Gennes-like renormalization [8]. Fitting the experimental data in that $Q$ regime leaving $\tau_{d}$ as a free parameter yields the values of $\tau_{d}$ included as filled circles in Fig. 3(b). Then, assuming that $\tau_{d}$ crosses over from incoherent pure diffusive behavior towards those times, the description of the experimental susceptibility in a wide $Q$ range by the model function is excellent (see Fig. 2), with total amplitude factors that follow $S(Q)$ perfectly [see Fig. 1(a)]. The resulting relative amplitudes of the processes are represented in Fig. 3(a). They are in excellent agreement with the hypothesis of the model. As expected, the maximum of the diffusivelike contribution takes place in the $Q$ range of the maximum of $S(Q)$. This contribution decreases at lower $Q$ values, becoming almost negligible at $Q \lesssim 0.8 \AA^{-1}$. The values obtained for these contributions from the analysis of the MD-simulation results (see SM [19]) agree with the experimental trend [see Fig. 3(a)]. Furthermore, the average characteristic time of the relaxational contribution

$$
\langle\tau\rangle=\int_{0}^{\infty} F_{R}(Q, t) d t=[1-A(Q)] \tau_{c}+A(Q) \tau_{d}
$$

is represented by the dashed-dotted line in Fig. 3(b). It nicely reflects how the structural relaxation crosses over from being dominated by the $Q$-independent collective mode in the low- $Q$ mesoscale to be mainly driven by diffusion at intermolecular scales.

The results obtained for 280 and $350 \mathrm{~K}$ follow the same trend with $Q$ (see SM [19]), although the relative amplitude of the effective $Q^{o}$ mode increases with temperature. The values of $\tau_{c}^{o}$ at the three different temperatures are included in the inset of Fig. 3(b). They agree rather well with those of the so-called "structural" time obtained from the analysis of the IXS measurements extrapolated to $Q=0$ [4] and with other characteristic times of water dynamics [see inset of Fig. 3(b) and the corresponding caption]. An Arrhenius fit of $\tau_{c}^{o}$ delivers an apparent activation energy of $\approx 4 \mathrm{Kcal} / \mathrm{mol}$, which is in the range of values from different low-frequency, and usually low-temperature, measurements: ultrasonic $(\approx 4 \mathrm{Kcal} / \mathrm{mol}$ [32]); hypersonic ( $\approx 4.2 \mathrm{Kcal} / \mathrm{mol}[33] ; 5.2 \mathrm{Kcal} / \mathrm{mol}[25])$; 
and time-resolved spectroscopy $(\approx 6 \mathrm{Kcal} / \mathrm{mol}[34])$ and inelastic ultraviolet $(\mathrm{UV})$ scattering $(\approx 5.5 \mathrm{Kcal} / \mathrm{mol}[26])$. These values are larger than that of a simple hydrogen bond (HB) $(\approx 2.8 \mathrm{Kcal} / \mathrm{mol})$. As discussed in Ref. [9] for $300 \mathrm{~K}$, at $t \sim 2$ ps $\left(\approx \tau_{c}^{o}\right)$ the mean-squared displacement of water molecules corresponds to the broad crossover range between local processes-involving breaking and forming of HBsand diffusion. This suggests that this kind of motions is behind the decorrelation of density fluctuations at low $Q$ ( $Q^{o}$ mode). We note that in the framework of a Mori-Zwanzig formalism the coherent time at $Q \rightarrow 0$ but not yet in the hydrodynamic range (i.e., $\tau_{c}^{o}$ in our terminology, can be expressed as $\tau_{c}^{o} \approx$ $\left(M_{L} / K_{B}\right) \tau_{\alpha}(0)$ [35], where $M_{L}$ is the longitudinal modulus, $K_{B}$ the bulk modulus, and $\tau_{\alpha}$ an $\alpha$-relaxation time associated with a typical $Q=0$ correlation function (e.g., viscoelastic response). Since for liquids $M_{L} / K_{B} \sim 1$ [35], we could also identify $\tau_{c}^{o}$ with the so-called $\alpha$-relaxation time of water. In the framework of a generalized hydrodynamic approach at the explored frequency range, $\tau_{c}^{o}$ could also be considered of the order of the time driving the generalized viscosity [36].

In summary, we have been able to measure separately the coherent and incoherent dynamical structure factor of heavy water in a wide $Q$ range. In the low- $Q$ mesoscale, the observed decorrelation of the collective fluctuations hardly depends on $Q$. In the crossover range towards intermolecular scales, where the structural relaxation is dominated by diffusion, the experimental results are nicely described by the convolution of a $Q$-independent mode and diffusion. Additional processes, sometimes invoked from IXS measurements, seem not to be needed. This work opens a way of approaching the unknown territory of coherent scattering, from meso- to intermolecular scales, not only in water under different conditions but also in H-bonded liquids, glass-forming liquids, and biological systems where water plays an important role. It also convincingly proves the power of recently implemented neutron-scattering polarization analysis capabilities, which can hugely impact the progress of microscopic dynamics investigations in fields such as soft matter or biology.

We thank Mark Devonport, Jon Bones, and Holly McPhillips of the ISIS Neutron and Muon Source (UKRI) for technical support during the LET experiment [37]. Authors from the Materials Physics Center (San Sebastián) gratefully acknowledge the financial support of the Eusko Jaurlaritza, code IT-1175-19, and the Ministerio de Economía y Competitividad code PGC2018-094548-B-I00 (MCIU/AEI/FEDER, UE).
[1] A. Rahman and F. H. Stillinger, Phys. Rev. A 10, 368 (1974).

[2] P. Bosi, F. Dupré, F. Menzingee, F. Sacchetti, and M. C. Spinelli, Lett. Nuovo Cimento (1971-1985) 21, 436 (1978).

[3] J. Teixeira, M. C. Bellissent-Funel, S. H. Chen, and B. Dorner, Phys. Rev. Lett. 54, 2681 (1985).

[4] A. Cunsolo, Adv. Condens. Matter Phys. 2015, 137435 (2015).

[5] J. S. Hansen, A. Kisliuk, A. P. Sokolov, and C. Gainaru, Phys. Rev. Lett. 116, 237601 (2016).

[6] E. Pontecorvo, M. Krisch, A. Cunsolo, G. Monaco, A. Mermet, R. Verbeni, F. Sette, and G. Ruocco, Phys. Rev. E 71, 011501 (2005).

[7] A. Cunsolo, C. N. Kodituwakku, F. Bencivenga, M. Frontzek, B. M. Leu, and A. H. Said, Phys. Rev. B 85, 174305 (2012).

[8] P. G. de Gennes, Physica 25, 825 (1959).

[9] A. Arbe, P. Malo de Molina, F. Alvarez, B. Frick, and J. Colmenero, Phys. Rev. Lett. 117, 185501 (2016).

[10] G. L. Squires, Introduction to Thermal Neutron Scattering (Cambridge University Press, Cambridge, UK, 1988).

[11] W. Heil, K. H. Andersen, R. Cywinski, H. Humblot, C. Ritter, T. W. Roberts, and J. R. Stewart, Nucl. Instrum. Methods Phys. Res., Sect. A 485, 551 (2002).

[12] J. R. Stewart, P. P. Deen, K. H. Andersen, H. Schober, J.-F. Barthélémy, J. M. Hillier, A. P. Murani, T. Hayes, and B. Lindenau, J. Appl. Crystallogr. 42, 69 (2009).

[13] F. Alvarez, J. Colmenero, R. Zorn, L. Willner, and D. Richter, Macromolecules 36, 238 (2003).

[14] A.-C. Genix, A. Arbe, F. Alvarez, J. Colmenero, W. Schweika, and D. Richter, Macromolecules 39, 3947 (2006).

[15] L. Temleitner, L. Pusztai, and W. Schweika, J. Phys.: Condens. Matter 19, 335207 (2007).

[16] T. Burankova, R. Hempelmann, A. Wildes, and J. P. Embs, J. Phys. Chem. B 118, 14452 (2014).
[17] G. Cassella, J. R. Stewart, G. M. Paternò, V. García-Sakai, M. Devonport, P. J. Galsworthy, R. I. Bewley, D. J. Voneshen, D. Raspino, and G. J. Nilsen, J. Phys.: Conf. Ser. 1316, 012007 (2019).

[18] W. Chen, S. Watson, Y. Qiu, J. A. Rodriguez-Rivera, and A. Faraone, Phys. B: Condens. Matter 564, 166 (2019).

[19] See Supplemental Material at http://link.aps.org/supplemental/ 10.1103/PhysRevResearch.2.022015, Sec. I, for details about the experimental method, which includes Refs. [17,20-22] and Fig. S1; Sec. II, for details about the MD simulations and the results obtained, which includes Refs. [4,9,23] and Figs. S2, S3, and S4; Sec. III, where the calculation of the susceptibility from the measured spectra is explained; Sec. IV, where the analysis of the incoherent dynamic structure factor is described, which includes Ref. [9] and Fig. S5; and Sec. V, where the results obtained from the analysis of the data at 280 and $350 \mathrm{~K}$ are presented. This section includes Ref. [24] and Figs. S6, S7, and $\mathrm{S} 8$.

[20] G. J. Nilsen, J. Košata, M. Devonport, P. J. Galsworthy, R. I. Bewley, D. J. Voneshen, R. Dalgliesh, and J. R. Stewart, J. Phys.: Conf. Ser. 862, 012019 (2017).

[21] J. Kosata, G. J. Nilsen, M. Devonport, R. I. Bewley, D. J. Voneshen, P. J. Galsworthy, D. Raspino, and J. R. Stewart, Physica B 551, 476 (2018).

[22] C. J. Beecham, S. Boag, C. Frost, T. J. McKetterick, J. R. Stewart, K. H. Andersen, P. M. Bentley, and D. Jullien, Phys. B (Amsterdam, Neth.) 406, 2429 (2011).

[23] Discovery Studio 3.0.0.10321, Accelrys Software, Inc.

[24] J. Qvist, H. Schober, and B. Halle, J. Chem. Phys. 134, 144508 (2011).

[25] A. Cunsolo and M. Nardone, J. Chem. Phys. 105, 3911 (1996).

[26] F. Bencivenga, A. Cimatoribus, A. Gessini, M. G. Izzo, and C. Masciovecchio, J. Chem. Phys. 131, 144502 (2009). 
[27] J. Qvist, C. Mattea, E. P. Sunde, and B. Halle, J. Chem. Phys. 136, 204505 (2012).

[28] P. H. Handle, L. Rovigatti, and F. Sciortino, Phys. Rev. Lett. 122, 175501 (2019).

[29] A. Arbe, J. Colmenero, and D. Richter, in Broadband Dielectric Spectroscopy, edited by F. Kremer and A. Schönhals (SpringerVerlag, Berlin/Heidelberg, 2003), p. 685.

[30] A. Arbe, U. Buchenau, L. Willner, D. Richter, B. Farago, and J. Colmenero, Phys. Rev. Lett. 76, 1872 (1996).

[31] A. Arbe, D. Richter, J. Colmenero, and B. Farago, Phys. Rev. E 54, 3853 (1996).
[32] W. M. Slie, A. R. Donfor Jr., and T. A. Litovitz, J. Chem. Phys. 44, 3712 (1966).

[33] J. Rouch, C. C. Lai, and S. H. Chen, J. Chem. Phys. 65, 4016 (1976).

[34] R. Torre, P. Bartolini, and R. Righini, Nature 428, 296 (2004).

[35] V. N. Novikov, K. S. Schweizer, and A. P. Sokolov, J. Chem. Phys. 138, 164508 (2013).

[36] J. P. Boon and S. Yip, Molecular Hydrodynamics (Dover, New York, 1980).

[37] 10.5286/ISIS.E.RB1910280. 